\title{
Two- and three-body resonances in the $\bar{K} N N-\pi \Sigma N$ system
}

\author{
N.V. Shevchenko ${ }^{1, a}$ \\ Nuclear Physics Institute, 25068 Řež, Czech Republic
}

\begin{abstract}
One of the most important characteristics of a two-body interaction is existence and properties of its bound states or resonances. When the interaction is used in a few- or many-body calculation they also strongly influence the properties of the few- or many-body system. We investigate the dependence of the three-body resonance properties in the $\bar{K} N N$ system on the different models of $\bar{K} N$ interaction, providing one or two poles for $\Lambda(1405)$ resonance. The $\bar{K} N N-\pi \Sigma N$ system is described by coupled-channel Faddeev equations in AGS form, our two-body $\bar{K} N-\pi \Sigma$ interaction models reproduce all existing experimental data on $K^{-} p$ scattering and $K^{-} p$ atom level shift.
\end{abstract}

\section{Introduction}

Kaonic atoms and, especially, possibility of kaonic nuclear clusters formation attracted large interest recently. Hadronic nuclei are useful tools for studying hadron-nucleon interactions and in-medium properties of hadrons. Among $\bar{K}$ - nuclear systems, the study of three-body "exotic" systems offers the advantage that Faddeev equations [1], exactly describing the dynamics of few particles, provide. The history of the lightest possible antikaon-nucleus system, $K^{-} p p$, started by theoretical prediction made in [2] with values of $48 \mathrm{MeV}$ and $61 \mathrm{MeV}$ for the binding energy and the decay width, respectively. The first experimental evidence for a bound state $K^{-} p p$ state was observed by FINUDA collaboration at DA $\Phi$ NE [3] through its decay into back-to-back $\Lambda$-proton pairs. The deduced binding energy $(115 \mathrm{MeV})$, but not the width $(67 \mathrm{MeV})$, differs considerably from the theoretical prediction of [2]. In [4] the first genuinely three-body $\bar{K} N N-\pi \Sigma N$ coupled-channel Faddeev calculation was performed in search for quasibound states in the $K^{-} p p$ system. Since that, many theoretical works and some experimental evidences appeared. The most recent information about current situation in $\bar{K}-$ nuclear field can be found, say, in [5].

One of the most important characteristics of a twobody interaction is the existence and properties of its bound states or resonances. When the interaction is used in a fewor many-body calculation they also strongly influence the properties of the few- or many-body system. For the basic for $\bar{K}$ - nuclear systems $\bar{K} N$ interaction $\Lambda(1405)$ is the resonance, which plays an important role.

\section{$2 \Lambda(1405)$ resonance and $\bar{K} N-\pi \Sigma$ system}

It is known, that $\bar{K} N$ is strongly coupled to the $\pi \Sigma$ channel through the $\Lambda(1405)$ resonance. However, already the na-

\footnotetext{
a e-mail: shevchenko@ujf.cas.cz
}

ture of the resonance is a question. A usual assumption is that it is a resonance in $\pi \Sigma$ and a quasi-bound state in $\bar{K} N$ channel. There is also an assumption suggested by a chiral model, that the bump, which is usually understood as $\Lambda(1405)$ resonance, is an effect of two poles (see e.g. [6]).

Experimental $\bar{K} N$ data are imprecise, old or controversial. The data on cross-sections of elastic and inelastic scattering with $K^{-} p$ in the initial state are old and have large errors, while threshold branching ratios of $K^{-} p$ scattering were measured more accurately. Another source of knowledge about $\bar{K} N$ is kaonic hydrogen atom. Several experiments were performed for measuring $1 s$ level shift caused by strong $\bar{K} N$ interaction. The two recent ones are KEK [7] and DEAR [8] results. More recent DEAR value of $1 s$ level shift and width significantly differs from the older KEK result. It has smaller errors, but is inconsistent with the scattering $K^{-} p$ data as was shown in $[9,10]$.

Our aim was to construct two versions of phenomenological coupled-channel $\bar{K} N-\pi \Sigma$ potential having one- or two-pole form for $\Lambda(1405)$ resonance and, if possible, simultaneously reproducing all other experimental data: the level shift and width of kaonic hydrogen $1 s$ level (KEK or DEAR values), $K^{-} p$ threshold branching ratios, elastic and inelastic $K^{-} p$ scattering. For proper description of kaonic hydrogen atom we directly included Coulomb interaction, which breaks isospin symmetry, into the calculations. One more isospin-breaking effect was using of physical masses.

The total potential acting in $\bar{K} N-\pi \Sigma$ system consists of Coulomb $V_{c}$ and strong $V_{s}$ parts

$$
V_{t o t}=V_{c}+V_{s} \text {. }
$$

The strong part $V_{s}$ of the potential is separable, in momentum representation it has a form:

$$
V_{I_{i}, I_{j}}^{\alpha \beta}\left(k^{\alpha}, k^{\prime \beta}\right)=\lambda_{I_{i}, I_{j}}^{\alpha \beta} g_{I_{i}}^{\alpha}\left(k^{\alpha}\right) g_{I_{j}}^{\beta}\left(k^{\prime \beta}\right) .
$$

Here $I_{i}$ is a (non-conserving) two-body isospin, $\alpha$ is a channel index: $\alpha=K(\bar{K} N$-channel) or $\pi(\pi \Sigma$-channel). Parameters $\lambda_{I_{i}, I_{j}}^{\alpha \beta}$ were fitted to reproduce $K^{-} p$ experimental data. 
For the one-pole form of $\Lambda(1405)$ we used Yamaguchi form-factors:

$$
g_{I_{i}}^{\alpha}\left(k^{\alpha}\right)=\frac{1}{\left(k^{\alpha}\right)^{2}+\left(\beta_{I_{i}}^{\alpha}\right)^{2}}
$$

for both particle channels. We assumed $\Lambda(1405)$ as a resonance in $\pi \Sigma$ and a quasi-bound state in $\bar{K} N$ channel. So, calculation of the pole position was done at physical sheet for $\bar{K} N$ and non-physical sheet for $\pi \Sigma$ channel.

For the two-pole case we assumed that there are two resonances in $\pi \Sigma$ channel. One of them, as before, originates from a bound state in $\bar{K} N$ channel, the other one from a resonance in $\pi \Sigma$ channel (with $\bar{K} N-\pi \Sigma$ coupling switched off). It is known that in one-channel case oneterm separable potential with Yamaguchi form-factors (3) and real strength parameters can not describe a resonance. So, in order to have a resonance in the uncoupled $\pi \Sigma$ channel, for two-pole $\Lambda(1405)$ case we used $\pi \Sigma$ form-factors in the following form:

$$
g_{I_{i}}^{\alpha}\left(k^{\alpha}\right)=\frac{1}{\left(k^{\alpha}\right)^{2}+\left(\beta_{I_{i}}^{\alpha}\right)^{2}}+\frac{s\left(\beta_{I_{i}}^{\alpha}\right)^{2}}{\left[\left(k^{\alpha}\right)^{2}+\left(\beta_{I_{i}}^{\alpha}\right)^{2}\right]^{2}},
$$

while for the $\bar{K} N$ channel here we used Yamaguchi formfactors (3). Both poles are once more situated at physical sheet for $\bar{K} N$ and non-physical sheet for $\pi \Sigma$ channel.

All physical values for data fitting were obtained by solving coupled-channel Lippmann-Schwinger equations with direct inclusion of Coulomb potential. Thus, the obtained resonances are dynamically generated ones. It is necessary to note, that energies of atomic (kaonic hydrogen $1 s$ level) and nuclear (one- and two-pole $\Lambda(1405)$ ) states were obtained from the same system of equations. Details of the formalism can be found in [11].

\subsection{Experimental data}

\subsubsection{Kaonic atom}

The $K^{-} p$ atomic $1 s$ level shift $\Delta E_{1 s}$ and width $\Gamma_{1 s}$ measured in the KEK experiment [7]

$$
\begin{aligned}
\Delta E_{1 s}^{K E K} & =-323 \pm 63 \pm 11 \mathrm{eV}, \\
\Gamma_{1 s}^{K E K} & =407 \pm 208 \pm 100 \mathrm{eV}
\end{aligned}
$$

and in the DEAR collaboration experiment [8]

$$
\begin{aligned}
\Delta E_{1 s}^{D E A R} & =-197 \pm 37 \pm 6 \mathrm{eV}, \\
\Gamma_{1 s}^{D E A R} & =249 \pm 111 \pm 30 \mathrm{eV}
\end{aligned}
$$

sufficiently differs from each other. We tried to reproduce one of these values within $1 \sigma$ interval. We would like to stress, that in our approach there is no intermediate reference to $K^{-} p$ scattering length when reproducing the level shift and the width. Of course, after finding a set of potential parameters we can calculate a strong scattering length, which exactly corresponds to the obtained $1 s$ level shift $\Delta E_{1 s}$ and width $\Gamma_{1 s}$.

\subsection{2 $\mathrm{K}^{-} \mathrm{p}$ scattering cross-sections}

Elastic and inelastic total cross sections with $K^{-} p$ in the initial state were measured in [13-17] (we did not take into consideration data from [18] with huge error bars). It is interesting, that there are no comments about non-existence of the total elastic cross-sections (except [9] and [10]) due to the singularity of the pure Coulomb transition matrix $T_{c}$, while the "total elastic" cross-sections are plotted by almost every author of $\bar{K} N$ interaction models. Having Coulomb interaction directly included into the calculations we could not ignore the problem. We defined "total elastic" $K^{-} p$ cross-section following the experimental works [13, 18]. Namely, the total cross-sections were obtained by integrating differential cross-sections in the $-1 \leq \cos \theta \leq$ 0.966 region instead of $-1 \leq \cos \theta \leq 1$.

\subsubsection{Threshold branching ratios}

Three threshold branching ratios of $K^{-} p$ scattering were measured rather accurately $[19,20]$. One of them is

$$
\gamma=\frac{\Gamma\left(K^{-} p \rightarrow \pi^{+} \Sigma^{-}\right)}{\Gamma\left(K^{-} p \rightarrow \pi^{-} \Sigma^{+}\right)}=2.36 \pm 0.04 .
$$

We oriented on the medium value

$$
\gamma=2.36
$$

The other two ratios $R_{c}$ and $R_{n}$, containing $K^{-} p \rightarrow \pi^{0} \Lambda$ cross-sections,

$$
\begin{aligned}
& R_{c}=\frac{\Gamma\left(K^{-} p \rightarrow \pi^{+} \Sigma^{-}, \pi^{-} \Sigma^{+}\right)}{\Gamma\left(K^{-} p \rightarrow \text { all inelastic channels }\right)}=0.664 \pm 0.011, \\
& R_{n}=\frac{\Gamma\left(K^{-} p \rightarrow \pi^{0} \Lambda\right)}{\Gamma\left(K^{-} p \rightarrow \text { neutral states }\right)}=0.189 \pm 0.015
\end{aligned}
$$

could not be used in a straightforward way because we did not include $\pi^{0} \Lambda$ channel directly into our calculations. However, the effect of the channel was effectively taken into account by allowing $\lambda_{\bar{K} N, \bar{K} N}^{1}$ parameter to have nonzero imaginary part (it significantly improved the agreement with the experimental cross-sections). It is easy to find from the measured $K^{-} p$ threshold branching ratios $\gamma$, $R_{c}$, and $R_{n}$, that relevant weight of $\pi^{0} \Lambda$ channel at $K^{-} p$ threshold among all possible inelastic channels is approximately equal to $6 \%$. So, the introduced imaginary part only slightly breaks unitarity in contrast to what happens when a one-channel complex $\bar{K} N$ potential is used, approximately accounting for the main inelastic $\pi \Sigma$ channel.

From existing $R_{c}$ and $R_{n}$ we constructed a new threshold branching ratio:

$$
R_{\pi \Sigma}=\frac{R_{c}}{1-R_{n}\left(1-R_{c}\right)}=0.709 \pm 0.011 .
$$

We tried to reproduce the medium value

$$
R_{\pi \Sigma}=0.709
$$



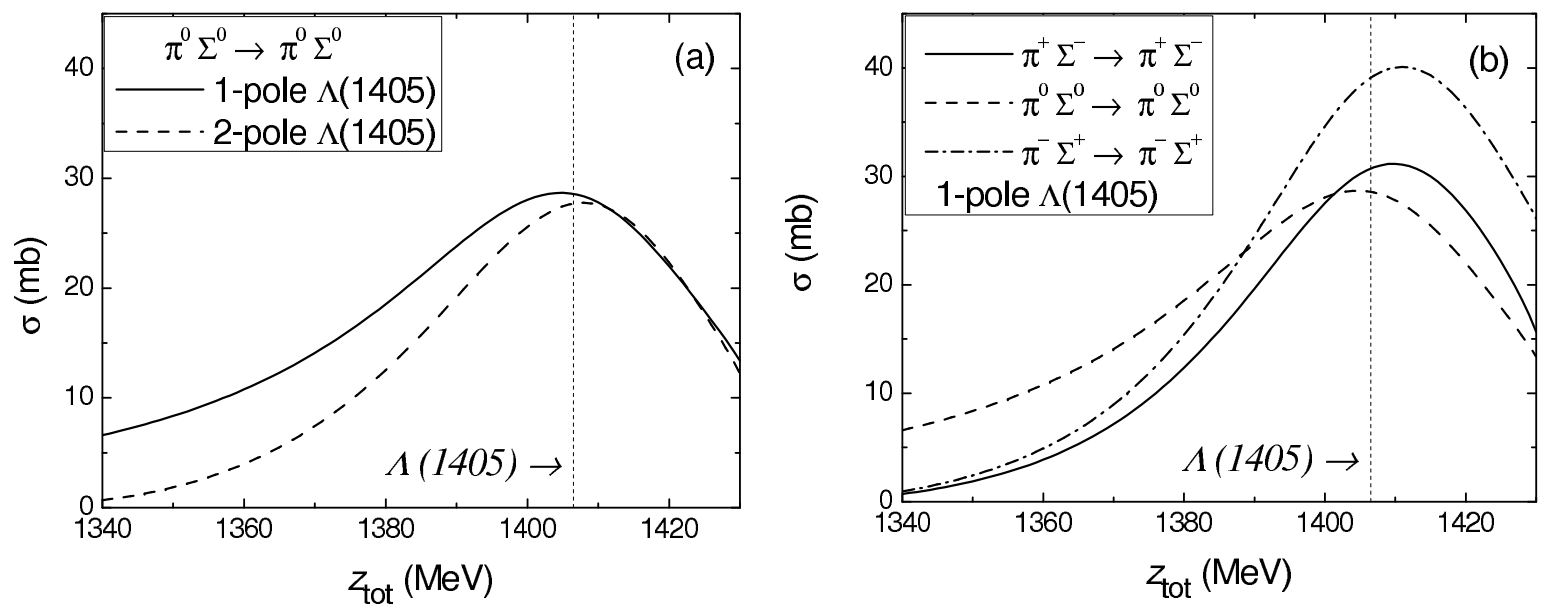

Fig. 1. The calculated pi-Sigma elastic cross-sections in different charge channels: (a) $\pi^{0} \Sigma^{0}$ (proportional to purely $I=0$ ) cross-sections given by the one-pole and two-pole $\Lambda(1405)$ potentials, (b) three charge channel cross-sections for the one-pole potential. The vertical line marks the medium PDG mass $M_{\Lambda}^{P D G}=1406.5 \mathrm{MeV}$ of the resonance [12].

\subsection{Two-body results}

The best sets of the obtained parameters for the one- and two-pole $\Lambda(1405)$ can be found in [11]. Some physical characteristics of the obtained potentials are shown in Table 1: pole positions $z_{1}$ and $z_{2}$ (obviously, $z_{2}$ exists in twopole variant of the potential only), $1 s$ kaonic hydrogen le-vel shift $\Delta E_{1 s}$ and width $\Gamma_{1 s}$. Threshold branching ratios $\gamma(10)$ and $R_{\pi \Sigma}$ (13) are reproduced exactly in both cases. Having complete set of potential parameters it is possible to calculate the strong $K^{-} p$ scattering length corresponding to the given $\Delta E_{1 s}$ and $\Gamma_{1 s}$ exactly. The $a_{K^{-}} p$ for both potentials are also shown in the Table 1 .

Table 1. Physical characteristics of the obtained one-pole and two-pole potentials: pole positions $z_{1}$ and $z_{2}$, level shift $\Delta E_{1 s}$ and width $\Gamma_{1 s}$ of kaonic hydrogen, and corresponding exact strong scattering length $a_{K^{-}}$. Threshold branching ratios (10) and (13) are reproduced exactly.

\begin{tabular}{ccc}
\hline \hline & one-pole $\Lambda(1405)$ & two-pole $\Lambda(1405)$ \\
\hline$z_{1}(\mathrm{MeV})$ & $1409-i 32$ & $1412-i 32$ \\
$z_{2}(\mathrm{MeV})$ & - & $1380-i 105$ \\
$\Delta E_{1 s}(\mathrm{eV})$ & -396 & -407 \\
$\Gamma_{1 s}(\mathrm{eV})$ & 370 & 476 \\
$a_{K^{-}}(\mathrm{fm})$ & $-1.07+i 0.59$ & $-1.08+i 0.76$ \\
\hline \hline
\end{tabular}

The first pole positions $z_{1}$ for both versions of the potential have close real parts and the same imaginary ones, however, all three numbers differ from the PDG data for mass and width of $\Lambda(1405)$ resonance:

$$
\begin{aligned}
M_{\Lambda}^{\mathrm{PDG}} & =1406.5 \pm 4.0 \mathrm{MeV}, \\
\Gamma_{\Lambda}^{\mathrm{PDG}} & =50.0 \pm 2.0 \mathrm{MeV} .
\end{aligned}
$$

The characteristics of the two poles $z_{1}$ and $z_{2}$ in the twopole $\Lambda(1405)$ version are the same as in chiral-based potentials: one of them has less mass and larger width, while the other is heavier with narrower width.

It is not absolutely clear, how to relate the obtained potentials to the shape of the $\Lambda(1405)$ resonance. The experimental shape of the resonance is deduced from missing mass experiments since direct $\pi \Sigma$ data are not available. However, their relation to the pole structure of the twobody $T$-matrix is not trivial and needs further investigation. Examples of this interpretation ambiguity are shown in Fig. 1, where elastic $\pi \Sigma$ cross-sections in different charge channels are plotted. It can be seen, that from the shapes of these curves it would be hard to deduce unambiguously the resonance parameters, given in Table 1. The important fact here is that cross-sections for different charged states in Fig. 1(b) are plotted for the one-pole version of the $\Lambda(1405)$ resonance. It proves, that difference in shapes and maxima hight, seen at the figure, has nothing in common with two-pole structure of the resonance in $\bar{K} N$ system.

Our results for the cross-sections with best set of the obtained parameters with one-pole and two-pole $\Lambda(1405)$ are presented in Fig. 2: the elastic $K^{-} p \rightarrow K^{-} p$ crosssection and inelastic $K^{-} p \rightarrow \bar{K}^{0} n, K^{-} p \rightarrow \pi^{+} \Sigma^{-}, K^{-} p \rightarrow$ $\pi^{-} \Sigma^{+}$, and $K^{-} p \rightarrow \pi^{0} \Sigma^{0}$ cross-sections are compared with existing experimental data [13-17]. It is seen, that both versions of the potential are equally good in describing the experimental data within the experimental errors. Due to this fact, unfortunately, it is not possible to give preference to one of the $\Lambda(1405)$ versions.

We plotted also the obtained parameters of kaonic hydrogen $\left(\Gamma_{1 s},\left|\Delta E_{1 s}\right|\right)$, shown in Table 1, together with the experimental $1 \sigma$ regions of KEK and DEAR results, see Fig. 3. It is seen, that obtained $\Delta E$ for the one-pole version is situated inside the KEK region, while for the twopole variant it is slightly outside. Both values are close to each other, they definitely prefer the largest values of KEK $|\Delta E|$. All our attempts to move the shift values to the DEAR region led to drastic worsening of the agreement 
EPJ Web of Conferences
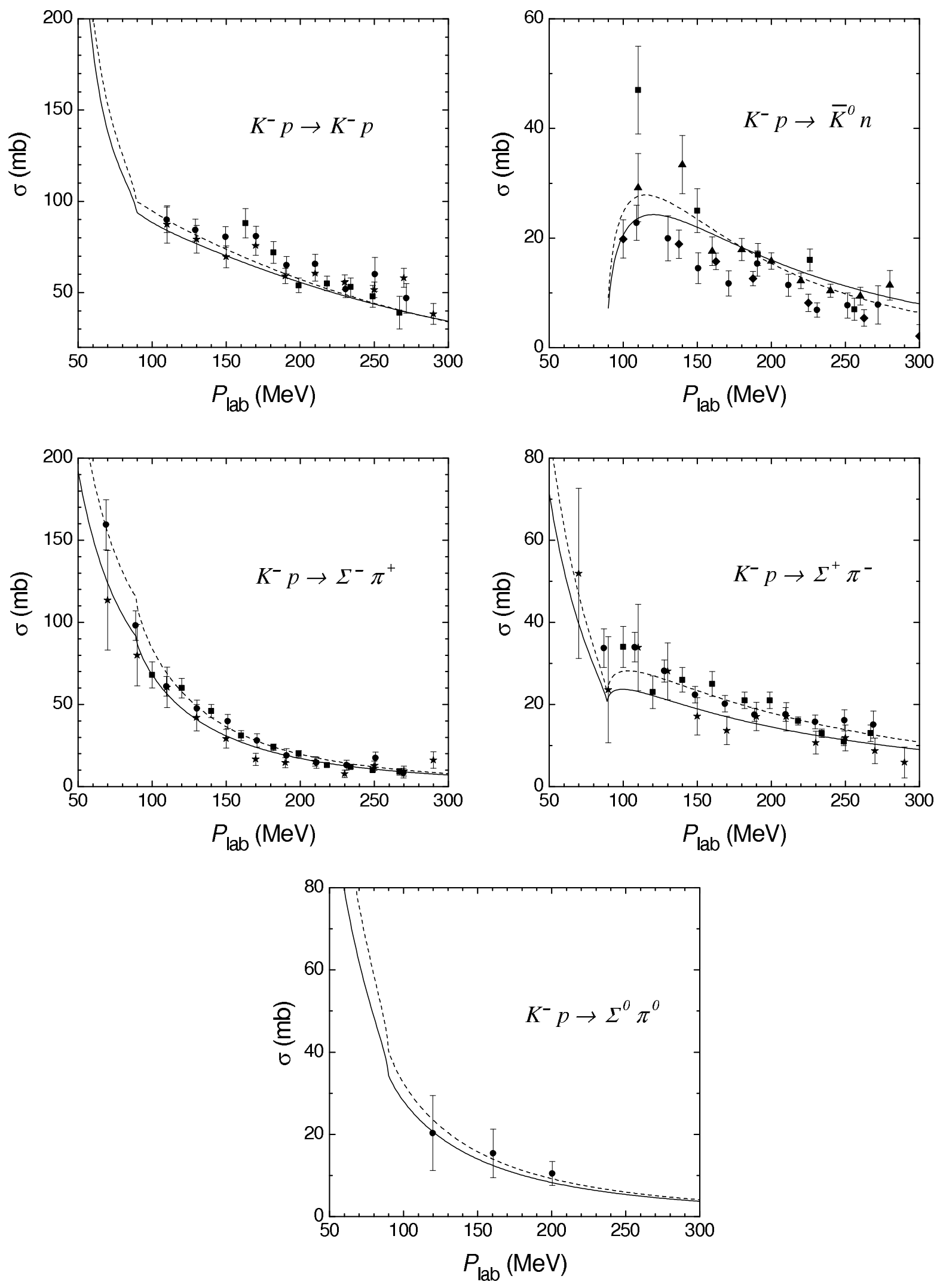

Fig. 2. Comparison of the obtained theoretical cross-sections (lines) with experimental data [13-17] (points). Solid lines: calculation with one-pole $\Lambda(1405)$ resonance, dashed lines: calculation with two-pole $\Lambda(1405)$ resonance. 
with the experimental cross-sections. From this fact we do the same conclusion as did authors of [10]: the DEAR data on kaonic hydrogen measurements are inconsistent with the existing scattering data.

As for the widths, both are situated inside KEK $1 \sigma$ limits, while the one-pole potential gives $\Gamma_{1 s}$ also inside DEAR, closely to its highest possible value. The important fact is that the obtained theoretical values of $\Gamma_{1 s}$ for the two versions of potentials have rather large difference. But, unfortunately, the accuracy of KEK results does not allow to make a unique selection between them.

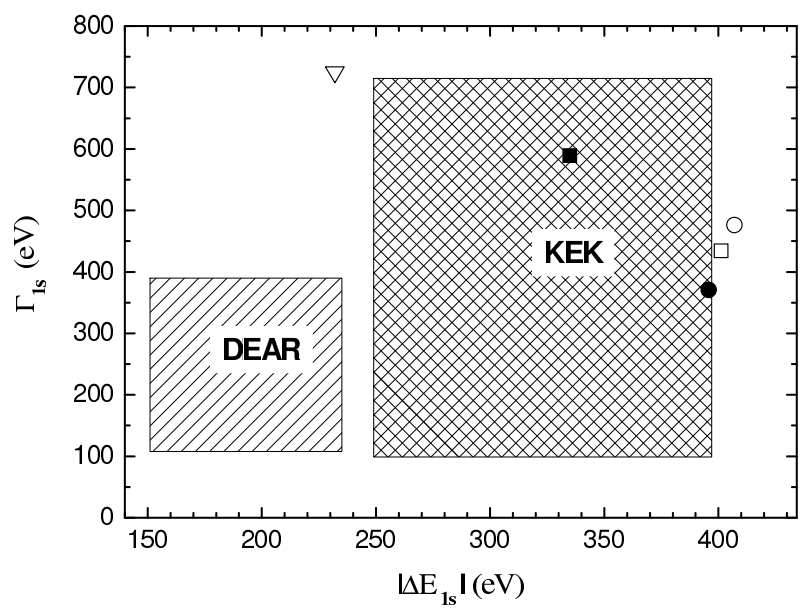

Fig. 3. DEAR and KEK $1 \sigma$ confidence region of kaonic hydrogen $1 s$ level shift $|\Delta E|$ (absolute value) and width $\Gamma$. The obtained theoretical results for the one-pole (solid circle) and two-pole (empty circle) variants of the potential are shown. The results of other theoretical models are also depicted: [9] (solid square), [10] (empty sqare), and [21] (empty triangle).

For comparison we plotted also the results of other theoretical models: [9], [10], and [21]. The first two values of $\left(\Gamma_{1 s},\left|\Delta E_{1 s}\right|\right)$ were obtained from the $K^{-} p$ scattering lengths using approximate formula from [22], which in what follows will be called "corrected Deser-Trueman formula" (cDT). The third value was calculated directly. The chiral potential [10], aiming to reproduce mainly the $K^{-} p$ scattering data, have the result (corresponding to the best $a_{K^{-} p}$ value in the full approach) impressively close to our, though the correctness of it is limited by the cDT formula accuracy.

The differences of the exact $K^{-} p$ level shifts and widths, obtained from our potentials, from results provided by approximate formulae are demonstrated in Table 2. The approximate Deser-Trueman (DT) [23] and corrected DeserTrueman [22] values for the shift and width were obtained using our exact scattering length given in the Table 1 . It is seen, that DT formula [23] gives very inaccurate result for both characteristics of kaonic atom: the absolute value of the level shift and the width are overestimated. The same result was obtained with several model one-channel complex $\bar{K} N$ potentials in [24]. The widely used nowadays
Table 2. Kaonic hydrogen $1 s$ level shift $\Delta E(\mathrm{eV})$ and width $\Gamma$ $(\mathrm{eV})$, corresponding to the obtained scattering length: exact ([11] and this work), derived from Deser-Trueman formula [23], and from corrected Deser-Trueman formula [22].

\begin{tabular}{lrr}
\hline \hline & one-pole $\Lambda(1405)$ & two-pole $\Lambda(1405)$ \\
\hline$\Delta E_{1 s}^{\text {exact }}$ & -396 & -407 \\
$\Gamma_{1 s}^{\text {exc }}$ & 370 & 476 \\
\hline$\Delta E_{1 s}^{D T}[23]$ & -441 & -445 \\
$\Gamma_{1 s}^{D T}[23]$ & 486 & 626 \\
\hline$\Delta E_{1 s}^{c D t}[22]$ & -395 & -411 \\
$\Gamma_{1 s}^{c D t}[22]$ & 338 & 434 \\
\hline \hline
\end{tabular}

cDT formula gives rather accurate result for the shift, but underestimates the width of $1 s$ level by $9-10 \%$.

We see that one- and two-pole versions of our potential reproduce experimental cross-sections equally well, by construction they exactly reproduce threshold branching ratios $\gamma$ and $R_{\pi \Sigma}$. The obtained values of the kaonic hydrogen level shift $\Delta E_{1 s}$ in both versions of $\Lambda(1405)$ resonance are close to each other. There is rather large, more than 100 $\mathrm{eV}$, difference between the $K^{-} p$ widths $\Gamma_{1 s}$. More precise experimental data on $K^{-} p$ atom, for example, from forthcoming SIDDHARTA experiment [25] could choose one of the variants of $\Lambda(1405)$ structure. However, at the moment it is not possible to draw conclusions about "nature" of $\Lambda(1405)$ resonance.

One possible way to clarify the situation is to perform a few- or many-body calculation using two versions of $\bar{K} N-$ $\pi \Sigma$ potential as an input. Having this in mind, we repeated our calculations of $\bar{K} N N-\pi \Sigma N$ system [4] looking for three-body poles in it. As before, coupled-channel Faddeev equations in AGS form [26] were solved.

\section{Three-body poles in $\bar{K} N N-\pi \Sigma N$ system}

\subsection{Coupled-channel AGS equations}

Original three-body Faddeev equations in the Alt-Grassberger-Sandhas (AGS) form [26]

$$
U_{i j}=\left(1-\delta_{i j}\right) G_{0}^{-1}+\sum_{k=1}^{3}\left(1-\delta_{i k}\right) T_{k} G_{0} U_{k j}
$$

define unknown operators $U_{i j}$, describing the elastic and re-arrangement processes $j+(k i) \rightarrow i+(j k)$. The inputs for the AGS system of equations (16) are two-body $T$ matrices, immersed into three-body space. The operator $G_{0}$ is the free three-body Green's function. Faddeev partition indices $i, j=1,2,3$ denote simultaneously an interacting pair and a spectator particle. When the initial state is known, as is usually assumed, the system (16) consists of three equations. Direct inclusion of the $\pi \Sigma N$ channel leads to the coupled-channels equations:

$$
U_{i j}^{\alpha \beta}=\delta_{\alpha \beta}\left(1-\delta_{i j}\right)\left(G_{0}^{\alpha}\right)^{-1}+\sum_{k, \gamma=1}^{3}\left(1-\delta_{i k}\right) T_{k}^{\alpha \gamma} G_{0}^{\gamma} U_{k j}^{\gamma \beta},
$$


where additional "particle" indices $\alpha, \beta=1,2,3$ are introduced in addition to the usual Faddeev indices, see Table 3.

Table 3. Interacting two-body subsystems for three partition $(i)$ and three 'particle' channel $(\alpha)$ indices. The interactions are further labelled by the two-body isospin values, entering the AGS equations with total three-body isospin $I=1 / 2$.

\begin{tabular}{cccc}
\hline \hline$i \backslash \alpha$ & $1(\bar{K} N N)$ & $2(\pi \Sigma N)$ & $3(\pi N \Sigma)$ \\
\hline 1 & $N N_{I=0,1}$ & $\sum N_{I=\frac{1}{2}, \frac{3}{2}}$ & $\sum N_{I=\frac{1}{2}, \frac{3}{2}}$ \\
2 & $\bar{K} N_{I=0,1}$ & $\pi N_{I=\frac{1}{2}, \frac{3}{2}}$ & $\pi \Sigma_{I=0,1}$ \\
3 & $\bar{K} N_{I=0,1}$ & $\pi \Sigma_{I=0,1}$ & $\pi N_{I=\frac{1}{2}, \frac{3}{2}}$ \\
\hline \hline
\end{tabular}

All operators in Eq. (17) act in this additional "particle" space. The two-body $T$-matrices have the following form:

$$
\begin{aligned}
T_{1} & =\left(\begin{array}{ccc}
T_{1}^{N N} & 0 & 0 \\
0 & T_{1}^{\Sigma N} & 0 \\
0 & 0 & T_{1}^{\Sigma N}
\end{array}\right), \\
T_{2} & =\left(\begin{array}{ccc}
T_{2}^{K K} & 0 & T_{2}^{K \pi} \\
0 & T_{2}^{\pi N} & 0 \\
T_{2}^{\pi K} & 0 & T_{2}^{\pi \pi}
\end{array}\right), \\
T_{3} & =\left(\begin{array}{ccc}
T_{3}^{K K} & T_{3}^{K \pi} & 0 \\
T_{3}^{\pi K} & T_{3}^{\pi \pi} & 0 \\
0 & 0 & T_{3}^{\pi N}
\end{array}\right),
\end{aligned}
$$

where $T_{i}^{N N}, T_{i}^{\pi N}$ and $T_{i}^{\Sigma N}$ are the usual one-channel twobody $T$-matrices in three-body space, describing $N N, \pi N$, and $\Sigma N$ interactions, respectively. The $T_{i}^{K K}, T_{i}^{\pi \pi}, T_{i}^{\pi K}$, and $T_{i}^{K \pi}$ are elements of the coupled-channel $T$-matrix, found in the previous section. The free Green's function is diagonal in channel indices: $G_{0}^{\alpha \beta}=\delta_{\alpha \beta} G_{0}^{\alpha}$, while the transition operators $U_{i j}^{\alpha \beta}$ have the most general form.

As in the previous calculation, we use a one-term separable form for $\bar{K} N-\pi \Sigma$ and $\Sigma N$ two-body potentials:

$$
V_{i, I}^{\alpha \beta}=\lambda_{i, I}^{\alpha \beta}\left|g_{i, I}^{\alpha}\right\rangle\left\langle g_{i, I}^{\beta}\right|,
$$

which leads to a separable form of $T$-matrices:

$$
T_{i, I}^{\alpha \beta}=\left|g_{i, I}^{\alpha}\right\rangle \tau_{i, I}^{\alpha \beta}\left\langle g_{i, I}^{\beta}\right|
$$

while for $N N$ interaction a two-term separable potential was used:

$$
V_{N N}=\sum_{m=1}^{2}\left|g_{m(N N)}\right\rangle \lambda_{m(N N)}\left\langle g_{m(N N)}\right|
$$

with

$$
T_{N N}=\sum_{m, n=1}^{2}\left|g_{m(N N)}\right\rangle \tau_{m n(N N)}\left\langle g_{n(N N)}\right| .
$$

The relations (22) and (24) allow to introduce new transition and kernel operators $X_{i j, I_{i} I_{j}}^{\alpha \beta}$ and $Z_{i j, I_{i} I_{j}}^{\alpha \beta}$, correspondingly, and rewrite the AGS system (17). The number of equations in the system on new unknown operators $X_{i j, I_{i} I_{j}}^{\alpha \beta}$ is defined by the number of possible form-factors $g$. Thus, in contrast to the previous calculation, after antisymmetrization our system consists of 10 equations due to the twoterm $T_{N N}$.

As before, to solve the homogeneous system we transform the integral equations into algebraic ones and then search for the complex energy at which the determinant of the kernel matrix becomes equal to zero. We are looking for a three-body pole, the real part of which is situated between the $\bar{K} N N$ and $\pi \Sigma N$ thresholds, corresponding to a resonance in the $\pi \Sigma N$ channel and a quasi-bound state (a bound state with non-zero width) in the $\bar{K} N N$ channel. Therefore, we work on the physical energy sheet of channel one and on an unphysical sheet of the second channel.

\subsection{Two-body input}

The separable potential (21), in momentum representation, has a form:

$$
V_{i, I_{i}}^{\alpha \beta}\left(k_{i}^{\alpha}, k_{i}^{\prime \beta}\right)=\lambda_{i, I_{i}}^{\alpha \beta} g_{i, I_{i}}^{\alpha}\left(k_{i}^{\alpha}\right) g_{i, I_{i}}^{\beta}\left(k_{i}^{\prime \beta}\right) .
$$

Not only basic $\bar{K} N-\pi \Sigma$ interaction was changed during the calculations, other two-body inputs were also improved. We once more neglected the $\pi N$ interaction since its dominant part is in the $(3,3) p$-wave channel.

\subsection{1 $\bar{K} N-\pi \Sigma$ potential}

We used one- and two-pole versions of the coupled-chan-nel potential described in Section II. Here we do not use Cou-lomb part of the interaction. Values for binding energies of the quasi-bound state in $\bar{K} N N$ system $(50-70 \mathrm{MeV})$ obtained in our calculations [4] show, that Coulomb interaction plays a minor role and safely can be omitted. Thus, only strong part $V_{s}$ of the obtained in Section II potential was used during tree-body calculation.

\subsection{2 $N N$ potential}

In comparison with our previous three-body calculations we used also a new $N N$ potential [27]. The potential has two terms:

$$
V_{N N}\left(k, k^{\prime}\right)=\sum_{i=1}^{2} g_{i(N N)}(k) \lambda_{i(N N)} g_{i(N N)}\left(k^{\prime}\right),
$$

it reproduces Argonne $N N v 18$ phase shifts and therefore has repulsion at short distances, see Fig. 4. Two versions of the potential ("A" and "B") differing by form-factors were used:

$$
\begin{aligned}
& g_{i(N N)}^{A}(k)=\sum_{m=1}^{2} \frac{\gamma_{i m}^{A}}{\left(\beta_{i m}^{A}\right)^{2}+k^{2}}, i=1,2 \\
& g_{1(N N)}^{B}(k)=\sum_{m=1}^{3} \frac{\gamma_{1 m}^{B}}{\left(\beta_{1 m}^{B}\right)^{2}+k^{2}}, g_{2(N N)}^{B}(k)=\sum_{m=1}^{2} \frac{\gamma_{2 m}^{B}}{\left(\beta_{2 m}^{B}\right)^{2}+k^{2}} .
\end{aligned}
$$


$19^{\text {th }}$ International IUPAP Conference on Few-Body Problems in Physics

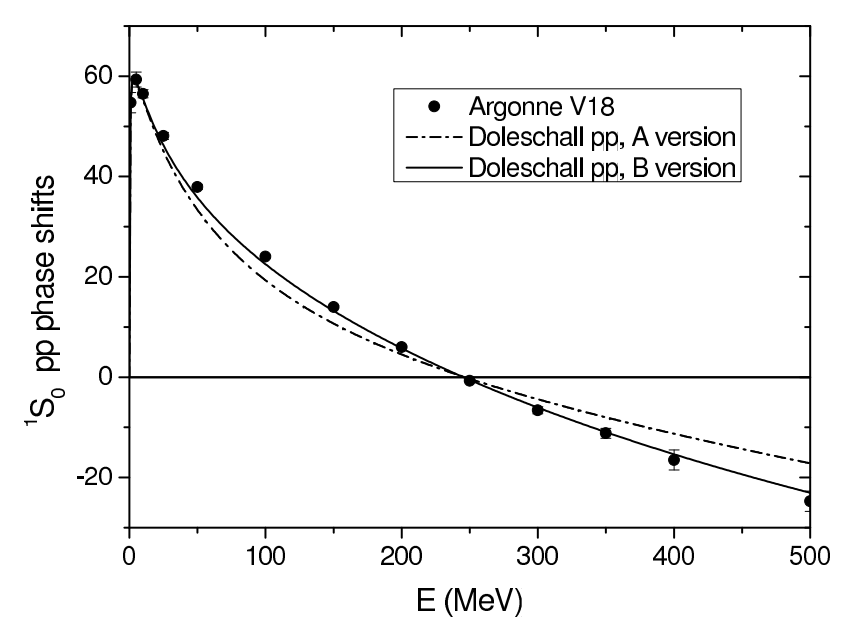

Fig. 4. ${ }^{1} S_{0}$ pp phase shifts for two versions of the $N N$ potential [27] (lines) in comparison with those of Argonne $v 18$ potential (solid circles).

The potential reproduces the following scattering length and effective radius:

$$
\begin{aligned}
& a^{A}(p p)=16.553 \mathrm{fm}, r_{\mathrm{eff}}^{A}(p p)=2.845 \mathrm{fm}, \\
& a^{B}(p p)=16.558 \mathrm{fm}, r_{\mathrm{eff}}^{B}(p p)=2.880 \mathrm{fm} .
\end{aligned}
$$

\subsection{3 $\Sigma N(-\Lambda N)$ interaction}

It was shown in [4], that this interaction has rather small influence on the three-body results. However, in order to be consistent we constructed new version of $\Sigma N$ interaction in such a way, that it reproduces existing experimental data [29-33]. As before, a separable potential (25) with Yamaguchi form-factors

$$
g_{I}^{\Sigma N}(k)=\frac{1}{k^{2}+\left(\beta_{I}^{\Sigma N}\right)^{2}}
$$

was used for the two isospin states.

The parameters of the $I=3 / 2 \Sigma N$ interaction were fitted to the $\Sigma^{+} p \rightarrow \Sigma^{+} p$ cross-sections. It is a one-channel interaction, so $\lambda_{I=3 / 2}$ parameter is real.

Another situation is in $I=1 / 2$ state because it is coupled with $\Lambda N$ channel. Due to this we started by constructing a coupled-channel potential of the $\Sigma N-\Lambda N$ interaction, were all $\lambda$ parameters are real. This coupled-channel $I=1 / 2 \Sigma N-\Lambda N$ together with $I=3 / 2 \Sigma N$ reproduces $\Sigma^{-} p \rightarrow \Sigma^{-} p, \Sigma^{-} p \rightarrow \Sigma^{0} n, \Sigma^{-} p \rightarrow \Lambda n$, and $\Lambda n \rightarrow \Lambda n$ cross-sections. After this we obtained an equivalent to the coupled-channel $\Sigma N-\Lambda N$ optical potential for one-channel $I=1 / 2 \Sigma N$ interaction. The scattering lengths for the different isospin states are:

$$
a_{3 / 2}^{\Sigma N}=1.95 \mathrm{fm}, \quad a_{1 / 2}^{\Sigma N}=-2.40+i 0.85 \mathrm{fm} .
$$

\subsection{Three-body results}

The preliminary results of the three-body calculations for the one-pole version of $\bar{K} N-\pi \Sigma$ potential:

$$
z_{N N(A)}^{1 \text { pole }}=-29.63-i 47.31 \mathrm{MeV}
$$

$$
z_{N N(B)}^{1 \text { pole }}=-30.90-i 47.38 \mathrm{MeV}
$$

and for the two-pole version:

$$
\begin{aligned}
& z_{N N(A)}^{2 p o l e}=-59.50-i 41.13 \mathrm{MeV} \\
& z_{N N(B)}^{2 p o l e}=-59.66-i 41.31 \mathrm{MeV}
\end{aligned}
$$

show very small difference between results obtained with "A" and "B" versions of $N N$ interaction. However, different pole structure of the $\bar{K} N-\pi \Sigma$ interaction seems having large influence on the three-body pole positions. This finding is opposite to what we have seen in two-body case, where all experimental data were reproduced by these two versions of $\bar{K} N-\pi \Sigma$ interaction indistinguishably well. More detailed calculation is in progress.

Acknowledgments. The work was supported by the Czech GA AVCR grant KJB100480801.

\section{References}

1. L.D. Faddeev, Soviet Phys. JETP 12, 1014 (1961); Mathematical aspects of the three-body problem in quantum scattering theory, Steklov Math. Institute 69 (1963).

2. T. Yamazaki, Y. Akaishi, Phys. Lett. B 535, 70 (2002).

3. M. Agnello et al., Phys. Rev. Lett. 94, 212303 (2005).

4. N.V. Shevchenko, A. Gal, J. Mareš, Phys. Rev. Lett. 98, 082301 (2007); N.V. Shevchenko, A. Gal, J. Mareš, J. Révai, Phys. Rev. C 76, 044004 (2007).

5. A. Gal, arXiv:0912.2214 [nucl-th].

6. J. A. Oller and U.-G. Meißner, Phys. Lett. B 500, 263 (2001); D. Jido et.al, Nucl. Phys. A 725, 181 (2003).

7. M. Iwasaki et al., Phys. Rev. Lett. 78, 3067 (1997); T.M. Ito et al., Phys. Rev. C 58, 2366 (1998).

8. G. Beer et al., Phys. Rev. Lett. bf 94, 212302 (2005).

9. B.Borasoy, R.Nißler, W.Weise, Phys. Rev. Lett. 94, 213401 (2005); Eur. Phys. J. A 25, 79 (2005).

10. B. Borasoy, U.-G. Meißner, R. Nißler, Phys. Rev. C 74, 055201 (2006).

11. J. Révai, N.V. Shevchenko, Phys. Rev. C 79, 035202 (2009).

12. C. Amsler et al. (Particle Data Group), Phys. Lett. B 667, 1 (2008).

13. M. Sakitt et al., Phys. Rev. 139, B719 (1965).

14. J.K. Kim, Phys. Rev. Lett. 14, 29 (1965); Columbia University Report, Nevis, 149 (1966); Phys. Rev. Lett. 19, 1074 (1967).

15. W. Kittel, G. Otter, and I. Wacek, Phys. Lett. 21, 349 (1966).

16. J. Ciborowski et al., J. Phys. G 8, 13 (1982).

17. D. Evans et al., J. Phys. G 9, 885 (1983).

18. W.E. Humphrey, R.R. Ross, Phys. Rev. 127, 1305 (1962).

19. D.N. Tovee et al., Nucl. Phys. B 33, 493 (1971).

20. R.J. Nowak et al., Nucl. Phys. B 139, 61 (1978).

21. A. Cieplý, J. Smejkal, Eur. Phys. J. A 34, 237 (2007).

22. U.-G. Meißner, U. Raha, A. Rusetsky, Eur. Phys. J. C 35, 349 (2004). 
23. S. Deser et al., Phys. Rev. 96, 774 (1954); T. L. Trueman, Nucl. Phys. 26, 57 (1961).

24. J. Révai, N. V. Shevchenko, Few-Body Syst. 42, 83 (2008).

25. www.lnf.infn.it $\rightarrow$ Nuclear Physics $\rightarrow$ SIDDHARTA.

26. E.O. Alt, P. Grassberger, W. Sandhas, Nucl. Phys. B 2, 167 (1967).

27. P. Doleschall, private communication.

28. R. B. Wiringa, V. G. J. Stoks, and R. Schiavilla, Phys. Rev. C 51, 38 (1995).

29. G. Alexander, U. Karshon, A. Shapira, G. Yekutieli, R. Engelmann, H. Filthuth, and W. Lughofer, Phys. Rev. 173, 1452 (1968).

30. B. Sechi-Zorn, B. Kehoe, J. Twitty, and R. A. Burnstein, Phys. Rev. 175, 1735 (1968).

31. F. Eisele, H. Filthuth, W. Fölisch, V. Hepp, E. Leitner, and G. Zech, Phys. Lett. B37, 204 (1971).

32. R. Engelmann, H. Filthuth, V. Hepp, and E. Kluge, Phys. Lett. 21, 587 (1966).

33. V. Hepp and M. Schleich, Z. Phys. 214, 71 (1968). 